\title{
A RESULT ON RICCI CURVATURE AND THE SECOND BETTI NUMBER*
}

\author{
JIANMING WAN $^{\dagger}$
}

\begin{abstract}
We prove that the second Betti number of a compact Riemannian manifold vanishes under certain Ricci curved restriction. As consequences we obtain an interesting curved restriction for compact Kähler-Einstein manifolds and a homology sphere theorem in $\operatorname{dim}=4,5$.
\end{abstract}

Key words. Ricci curvature, Betti number.

AMS subject classifications. Primary 53C20; Secondary 53C25.

1. Introduction. The study of relation between curvature and topology is the central topic in Riemannian geometry. One of the strong tool is Bochner technique. It plays a very important role in understanding relation between curvature and Betti numbers. The first result in this field is Bochner's classical result (c.f. [6])

TheOREM 1.1. (Bochner 1946) Let $M$ be a compact Riemannian manifold with Ricci curvature Ric R $_{M}>0$. Then the first Betti number $b_{1}(M)=0$.

Berger investigated that in what case the second Betti number vanishes. He proved the following (c.f. [1], also see [2] theorem 2.8)

THEOREM 1.2. (Berger) Let $M$ be a compact Riemannian manifold of dimension $n \geq 5$. Suppose that $n$ is odd and the sectional curvature satisfies that $\frac{n-3}{4 n-9} \leq K_{M}<$ 1. Then the second Betti number $b_{2}(M)=0$.

Consider a different curvature condition, Micallef and Wang proved (c.f. [4], also see [2] theorem 2.7)

THEOREM 1.3. (Micallef-Wang) Let $M$ be a compact Riemannian manifold of dimension $n \geq 4$. Suppose that $n$ is even and $M$ has positive isotropic curvature. Then the second Betti number $b_{2}(M)=0$.

Here positive isotropic curvature means, for any four othonormal vectors $e_{1}, e_{2}, e_{3}, e_{4} \in T_{p} M$, the curvature tensor satisfies

$$
R_{1313}+R_{1414}+R_{2323}+R_{2424}>2\left|R_{1234}\right| \text {. }
$$

Recall that the Rauch-Berger-Klingenberg's sphere theorem (c.f. [1]) states that a simple connected compact Riemannian manifold is homeomorphic to a sphere if the sectional curvatures lie in $\left(\frac{1}{4}, 1\right]$. A generalization of sphere theorem (dues to MicallefMoore c.f. [5]) says that a compact simply connected Riemannian manifold with positive isotropic curvature is a homotopy sphere. Hence with the help of Poincare conjecture it is homeomorphic to a sphere. From the two theorems we know that theorems 1.2 and 1.3 can not cover too many examples.

In this note we shall use Ricci curvature to give a relaxedly sufficient condition for the second Betti number vanishing. Our main result is

\footnotetext{
${ }^{*}$ Received June 27, 2012; accepted for publication April 17, 2013.

†Department of Mathematics, Northwest University, Xi'an 710127, China (wanj_m@aliyun.com; wan@nwu.edu.cn). The research is supported by the National Natural Science Foundation of China N0.11301416.
} 
THEOREM 1.4. Let $M$ be a compact Riemannian manifold. The dimension $\operatorname{dim}(M)=2 m$ or $2 m+1$. Let $\bar{k}$ (resp. $\underline{k}$ ) be the maximal (resp. minimal) sectional curvature of $M$. If the Ricci curvature of $M$ satisfies that

$$
\operatorname{Ric}_{M}>\bar{k}+\frac{2 m-2}{3}(\bar{k}-\underline{k}),
$$

then the second Betti number $b_{2}(M)=0$.

Particularly, if $M$ is a compact Riemannian manifold with nonnegative sectional curvature, then the second Betti number vanishes provided

$$
\operatorname{Ric}_{M}>\frac{2 m+1}{3} \bar{k}
$$

Note that there is no dimensional restriction in theorem 1.4.

Any compact Kähler manifold does not satisfy (1.1) since it has $b_{2} \geq 1$.

The condition 1.1 is a Ricci pinching condition. We mention that several other Ricci pinching type theorems obtained by $\mathrm{Gu}$ and $\mathrm{Xu}$ (c.f. [3] [7], ).

As an immediate consequence, we obtain a curvature restriction for special Einstein manifolds.

Corollary 1.5. Let $M$ be a compact Einstein manifold with nonzero second Betti number. Then the Ricci curvature satisfies

$$
R i c \leq \bar{k}+\frac{2 m-2}{3}(\bar{k}-\underline{k}) .
$$

In addition, if the sectional curvature is nonnegative, one must have

$$
R i c \leq \frac{2 m+1}{3} \bar{k}
$$

Particularly (1.3) holds for any compact Kähler-Einstein manifold.

REMARK 1.6. 1) The condition (1.1) implies that the maximal sectional curvature $\bar{k}>0$ : If $\bar{k} \leq 0$, then

$$
\bar{k} \geq R i c_{M}>\bar{k}+\frac{2 m-2}{3}(\bar{k}-\underline{k})
$$

We get $\bar{k}<\underline{k}$. This is a contradiction.

2) Since $\bar{k}>0$, of course (1.1) implies $\operatorname{Ric}_{M}>0$.

3) If the minimal sectional curvature $\underline{k}<0$. Since $\bar{k}>0$. If $\operatorname{dim}(M)=2 m+1$, from

$$
2 m \bar{k} \geq R i c_{M}>\bar{k}+\frac{2 m-2}{3}(\bar{k}-\underline{k}),
$$

one has

$$
\bar{k}>\frac{2 m-2}{4 m-1}|\underline{k}| .
$$

Similarly

$$
\bar{k}>\frac{1}{2}|\underline{k}|
$$


provided $\operatorname{dim}(M)=2 m$.

We use theorem 1.4 to test some simple examples.

EXAmple 1.7. 1) The space form $S^{n}, \bar{k}=\underline{k}=1$, Ric $=n-1=\bar{k}$ for $n=2$ and Ric $=n-1>\bar{k}$ for $n \neq 2, b_{2}\left(S^{2}\right)=1$ and $b_{2}\left(S^{n}\right)=0$ for $n \neq 2$.

2) $S^{2} \times S^{2}$ with product metric, $\bar{k}=1, \underline{k}=0$, Ric $=1<\bar{k}+\frac{2 n-2}{3}(\bar{k}-\underline{k})$, $b_{2}\left(S^{2} \times S^{2}\right)=2$.

3) $S^{m} \times S^{m}, m>4$ with product metric, $\bar{k}=1, \underline{k}=0$, Ric $=m-1>\frac{2 m+1}{3} \bar{k}$, $b_{2}=0$.

4) $\mathbb{C P}^{n}$ with Fubini-Study metric, $\bar{k}=4, \underline{k}=1$, Ric $=2 n+2=\bar{k}+\frac{2 n-2}{3}(\bar{k}-\underline{k})$, $b_{2}\left(\mathbb{C P}^{n}\right)=1$.

From the examples we know that the inequality (1.1) is sharp.

The proof of theorem 1.4 is also based on Bochner technique. But comparing with Berger and Micallef-Wang's results, we consider a different side. This allows us get a uniform result (without dimensional restriction).

\section{Proof of the theorem.}

2.1. Bochner formula. Let $M$ be a compact Riemannian manifold. Let

$$
\Delta=d \delta+\delta d
$$

be the Hodge-Laplacian, where $d$ is the exterior differentiation and $\delta$ is the adjoint to $d$.

Let $\varphi \in \Omega^{k}(M)$ be a smooth $k$-form. Then we have the well-known Weitzenböck formula (c.f. [6])

$$
\Delta \varphi=\sum_{i} \nabla_{v_{i} v_{i}}^{2} \varphi-\sum_{i, j} \omega^{i} \wedge i\left(v_{j}\right) R_{v_{i} v_{j}} \varphi,
$$

here $\nabla_{X Y}^{2}=\nabla_{X} \nabla_{Y}-\nabla_{\nabla_{X} Y}$ and $R_{X Y}=-\nabla_{X} \nabla_{Y}+\nabla_{Y} \nabla_{X}+\nabla_{[X, Y]}$. The $\left\{v_{i}, 1 \leq\right.$ $i \leq n\}$ are the local orthonormal vector fields and $\left\{\omega_{i}, 1 \leq i \leq n\right\}$ are the duality.

A $k$-form $\varphi$ is called harmonic if $\Delta \varphi=0$.

The famous Hodge theorem states that the de Rham cohomology $H_{d R}^{k}(M)$ is isomorphic to the space spanned by $k$-harmonic forms.

Let $\varphi=\sum_{i, j} \varphi_{i j} \omega^{i} \wedge \omega^{j}$ be a harmonic 2-form. By (2.1), under the normal frame we can get (c.f. [2] or [1])

$$
\Delta \varphi_{i j}=\sum_{k}\left(R i c_{i k} \varphi_{k j}+R i c_{j k} \varphi_{i k}\right)-2 \sum_{k, l} R_{i k j l} \varphi_{k l}
$$

where $R_{i j k l}=\left\langle R\left(v_{i}, v_{j}\right) v_{k}, v_{l}\right\rangle$ is the curvature tensor and $R i c_{i j}=\sum_{k}\left\langle R\left(v_{k}, v_{i}\right) v_{k}, v_{j}\right\rangle$ is the Ricci tensor.

So we have

$$
\begin{aligned}
\Delta|\varphi|^{2} & =2 \sum_{i, j} \varphi_{i j} \Delta \varphi_{i j}+2 \sum_{i, j} \sum_{k}\left(v_{k} \varphi_{i j}\right)^{2} \\
& \geq 2 \sum_{i, j} \varphi_{i j} \Delta \varphi_{i j} \\
& \triangleq 2 F(\varphi)
\end{aligned}
$$


Note that by (2.1) one has the global form of above formula

$$
0=-\langle\Delta \varphi, \varphi\rangle=\sum_{i}\left|\nabla_{v_{i}} \varphi\right|^{2}+\left\langle\sum_{i, j} \omega^{i} \wedge i\left(v_{j}\right) R_{v_{i} v_{j}} \varphi, \varphi\right\rangle-\frac{1}{2} \Delta|\varphi|^{2} .
$$

The $F(\varphi)$ is just the curvature term $\left\langle\sum_{i, j} \omega^{i} \wedge i\left(v_{j}\right) R_{v_{i} v_{j}} \varphi, \varphi\right\rangle$.

2.2. Proof of Theorem 1.4. By Hodge theorem, we only need to show that every harmonic 2 -form vanishes.

Case 1: Assume $\operatorname{dim}(M)=2 m$. For any $p \in M$, we can choose an orthonormal basis $\left\{v_{1}, w_{1}, \ldots, v_{m}, w_{m}\right\}$ of $T_{p} M$ such that $\varphi(p)=\sum_{\alpha} \lambda_{\alpha} v_{\alpha}^{*} \wedge w_{\alpha}^{*}$ (for instance c.f. [1] or [2]). Here $\left\{v_{\alpha}^{*}, w_{\alpha}^{*}\right\}$ is the dual basis. Then

$$
F(\varphi)=\sum_{\alpha=1}^{m} \lambda_{\alpha}^{2}\left[\operatorname{Ric}\left(v_{\alpha}, v_{\alpha}\right)+\operatorname{Ric}\left(w_{\alpha}, w_{\alpha}\right)\right]-2 \sum_{\alpha, \beta=1}^{m} \lambda_{\alpha} \lambda_{\beta} R\left(v_{\alpha}, w_{\alpha}, v_{\beta}, w_{\beta}\right)
$$

The term

$$
\begin{aligned}
& -2 \sum_{\alpha, \beta=1}^{m} \lambda_{\alpha} \lambda_{\beta} R\left(v_{\alpha}, w_{\alpha}, v_{\beta}, w_{\beta}\right) \\
= & -2 \sum_{\alpha \neq \beta} \lambda_{\alpha} \cdot \lambda_{\beta} \cdot R\left(v_{\alpha}, w_{\alpha}, v_{\beta}, w_{\beta}\right)-2 \sum_{\alpha=1}^{m} \lambda_{\alpha}^{2} R\left(v_{\alpha}, w_{\alpha}, v_{\alpha}, w_{\alpha}\right) \\
\geq & -\frac{4}{3}(\bar{k}-\underline{k}) \sum_{\alpha \neq \beta}\left|\lambda_{\alpha}\right| \cdot\left|\lambda_{\beta}\right|-2 \bar{k} \sum_{\alpha=1}^{m} \lambda_{\alpha}^{2} \\
\geq & -\frac{2}{3}(\bar{k}-\underline{k}) \sum_{\alpha \neq \beta}\left(\lambda_{\alpha}^{2}+\lambda_{\beta}^{2}\right)-2 \bar{k}|\varphi|^{2} \\
= & -\frac{2}{3}(\bar{k}-\underline{k})(2 m-2)|\varphi|^{2}-2 \bar{k}|\varphi|^{2} \\
= & -2\left[\bar{k}+\frac{2 m-2}{3}(\bar{k}-\underline{k})\right]|\varphi|^{2} .
\end{aligned}
$$

The first " $\geq "$ follows from Berger's inequality (c.f. [1]): For any orthonormal 4frames $\left\{e_{1}, e_{2}, e_{3}, e_{4}\right\}$, one has

$$
\left|R\left(e_{1}, e_{2}, e_{3}, e_{4}\right)\right| \leq \frac{2}{3}(\bar{k}-\underline{k}) .
$$

On the other hand, by the condition (1.1) we have

$$
\sum_{\alpha=1}^{m} \lambda_{\alpha}^{2}\left[\operatorname{Ric}\left(v_{\alpha}, v_{\alpha}\right)+\operatorname{Ric}\left(w_{\alpha}, w_{\alpha}\right)\right] \geq 2\left[\bar{k}+\frac{2 m-2}{3}(\bar{k}-\underline{k})\right]|\varphi|^{2},
$$

the equality holds if and only if $\varphi(p)=0$.

This leads to

$$
F(\varphi) \geq 0
$$

with equality if and only if $\varphi(p)=0$. Since

$$
\int_{M} F(\varphi) \leq \frac{1}{4} \int_{M} \Delta|\varphi|^{2}=0
$$


we get

$$
F(\varphi) \equiv 0
$$

Thus the harmonic 2-form $\varphi \equiv 0$.

Case 2: If $\operatorname{dim}(M)=2 m+1$. For any $p \in M$, we also can choose an orthonormal basis $\left\{u, v_{1}, w_{1}, \ldots, v_{m}, w_{m}\right\}$ of $T_{p} M$ such that $\varphi(p)=\sum_{\alpha} \lambda_{\alpha} v_{\alpha}^{*} \wedge w_{\alpha}^{*}$ (c.f. [1] or [2]). We also have

$$
F(\varphi)=\sum_{\alpha=1}^{m} \lambda_{\alpha}^{2}\left[\operatorname{Ric}\left(v_{\alpha}, v_{\alpha}\right)+\operatorname{Ric}\left(w_{\alpha}, w_{\alpha}\right)\right]-2 \sum_{\alpha, \beta=1}^{m} \lambda_{\alpha} \lambda_{\beta} R\left(v_{\alpha}, w_{\alpha}, v_{\beta}, w_{\beta}\right) .
$$

Thus the argument is same to the even dimensional case.

This completes the proof of the theorem.

\section{Sphere theorem in $\operatorname{dim} 4$ and 5 .}

TheOREM 3.1. Let $M$ be a compact Riemannian manifold. $\operatorname{dim} M=4$ or 5 . If

$$
\operatorname{Ric}_{M}>\frac{5 \bar{k}-2 \underline{k}}{3}
$$

then $M$ is a real homology sphere, i.e. $b_{i}(M)=0$ for $1 \leq i \leq \operatorname{dim} M-1$.

Proof. Since Ric $M>0$, from theorem 1.1 we know that $b_{1}(M)=0$. Theorem 1.4 implies that $b_{2}(M)=0$. With the help of Poincare duality, we obtain the theorem.

Finally we metion a differential sphere theorem for Ricci curvature obtained by $\mathrm{Gu}$ and $\mathrm{Xu}$ ( c.f. [3] theorem D).

THEOREM 3.2. Let $M$ be a simple conncted compact Riemannian n-manifold. If

$$
\operatorname{Ric}_{M}>\left(n-\frac{11}{5}\right) \bar{k},
$$

then $M$ is diffeomorphic to $S^{n}$.

\section{REFERENCES}

[1] M. Berger, Sur quelques variétés riemaniennes suffisamment pincées, Bull. Soc. Math. France, 88 (1960), pp. 57-71.

[2] S. Brendle And R. Schoen, Sphere theorems in geometry, Surveys in differential geometry. Vol. XIII. Geometry, analysis, and algebraic geometry: forty years of the Journal of Differential Geometry, pp. 49-84, Surv. Differ. Geom., 13, Int. Press, Somerville, MA, 2009.

[3] J. Gu AND H. XU, The sphere theorems for manifolds with positive scalar curvature, J. Differential Geom., 92:3 (2012), pp. 507-545.

[4] M. Micallef And M. Wang, Metrics with nonnegative isotropic curvature, Duke Math. J., $72: 3$ (1993), pp. 649-672.

[5] M. Micallef And J. D. Moore, Minimal two-spheres and the topology of manifolds with positive curvature on totally isotropic two-planes, Ann. of Math., 127 (1988), pp. 199-227.

[6] H. Wu, The Bochner technique in differential geometry, Math. Rep., 3:2 (1988), i-xii and pp. $289-538$.

[7] H. XU AND J. Gu, The differentiable sphere theorem for manifolds with positive Ricci curvature, Proc. Amer. Math. Soc., 140:3 (2012), pp. 1011-1021. 
